\title{
KINEMATICS OF REACHING AND GRASPING WITH A TOOL
}

\author{
Bin Zheng ${ }^{1}$, Christine L. MacKenzie ${ }^{2}$ \\ ${ }^{1}$ Legacy Health System, Portland, Oregon ${ }^{2}$ Simon Fraser University, Burnaby, British Columbia, Canada
}

\begin{abstract}
Kinematics of reaching and grasping are observed for prehension performed by the hand (natural prehension) and with a simple grasper held in the hand (remote prehension). Remote prehension is executed with a longer movement time, lower movement speed, extended deceleration phase and a relatively larger peak aperture compared to natural prehension. The kinematic changes in remote prehension are more pronounced when adults reached and grasped an object placed on a narrow than a wide base. Results suggested that the indirect and incomplete proprioception and sensorimotor integration with tool use are the main problems for movement control in remote manipulation. Implications of this study are discussed for design of tools and a safe work environment for tool use.
\end{abstract}

\section{INTRODUCTION}

Tool use is a fundamental activity of human kind. However, we have little understanding of the processes underlying tool use by human beings. When a tool is added to the motor system, it will cause humans to develop a new mode of movement coordination. The study uses prehension as a standard movement to examine the coordination built between the hand and the tool.

Prehension is a common movement that includes two components - reaching and grasping. Each responds separately to different object properties and they are coordinated with each other in many aspects (Jeannerod, 1981, 1984). Jeannerod proposed a central mechanism for controlling the timing of these two components (Jeannerod, 1981, 1984). However, Wing argued the coordination is built on a spatial aspect (Wing, Turton, \& Fraser, 1986). Lately, scientists notice the association between reaching and grasping is not fixed but subject to changes of task requirement (Jeannerod, 1988; Marteniuk, Leavitt, \& MacKenzie, 1990).

This study investigates the kinematics of reaching and grasping in prehension performed by the hand and by holding a grasper in hand. For simplification, we use "natural" and "remote" to name prehension performed by the hand directly or by holding a grasper in the hand. At least two aspects need to be taken into the consideration when controlling a tool - the mechanical properties and the perception difficulties caused by the tool use.

When holding a grasper, the hinge location that alters the aperture ratio may have a significant impact on the aperture profile of grasping. The length of the grasper may modify the velocity profile for reaching. Tool use also adds difficulty for sensory perception of a movement. Lack of direct proprioceptive information is a major problem. It is true that vision will take a role for information searching and compensate the loss of proprioception in the case of tool use (Driver \& Spence, 2000; Maravita, Spence, \& Driver, 2003; Maravita, Spence, Kennett, \& Driver, 2002). However, the ability for visual compensation is moderate. The movement accuracy deteriorates significantly due to the deprivation of proprioception on the movement segments (Rothwell et al., 1982; Taub, 1976). In cases of performing a task requiring a high level of precision with a tool, the impact of insufficient proprioception on task performance may be evident.

In this study, the accuracy requirement was introduced by changing the diameter of the base which supports the object. We predicted that reaching and grasping an object placed on a narrow base would lengthen the movement time and deceleration phase compared to the object on the wide base; the difference between the wide and narrow bases would be more pronounced in remote than natural prehension.

The grasper used in this study had a pivot in the middle of the tool (length ratio $=1$ ), which minimized the impact of mechanical properties of the tool on task performance. We predicted that subjects would open the hand wider in remote prehension due to the limited proprioceptive feedback from the tool compared to natural prehension.

Tasks were required to be performed by the preferred and the non-preferred hand of the subjects. The independent variable of performing was primarily set for an interrelated study on the degrees-of-freedom control of tool use. In this study, the lateralization on manual movement provides us with an opportunity to examine the impact of motor skills on prehensile performance. Movement skills are believed to be more developed on the side of the preferred than the nonpreferred hand (Elliott, Roy, \& Goodman, 1993).

\section{METHODS}

Twelve university students who were naive to the purpose of the study participated in the study. Ethical approval was obtained from Simon Fraser University.

The task was to reach, grasp, and lift up a dowel from either a wide $(5 \mathrm{~cm})$ or a narrow $(1 \mathrm{~cm})$ base using the preferred and non-preferred hand with and without holding a simple grasper. The graspers used in the experiment were modified from common kitchen tongs. The handle and the object-contact ends of the tongs were cut off; the tongs became a simple device with two straight metal pieces $(0.5 \mathrm{~cm}$ in diameter) linked by a hinge in the middle. The total length of the grasper was $12.5 \mathrm{~cm}$. When used, the grasper was attached to the pad of the thumb and the index finger by surgical tape. The contact length between the grasper and hand was about $1 \mathrm{~cm}$, held constant over all subjects (Figure 1). 


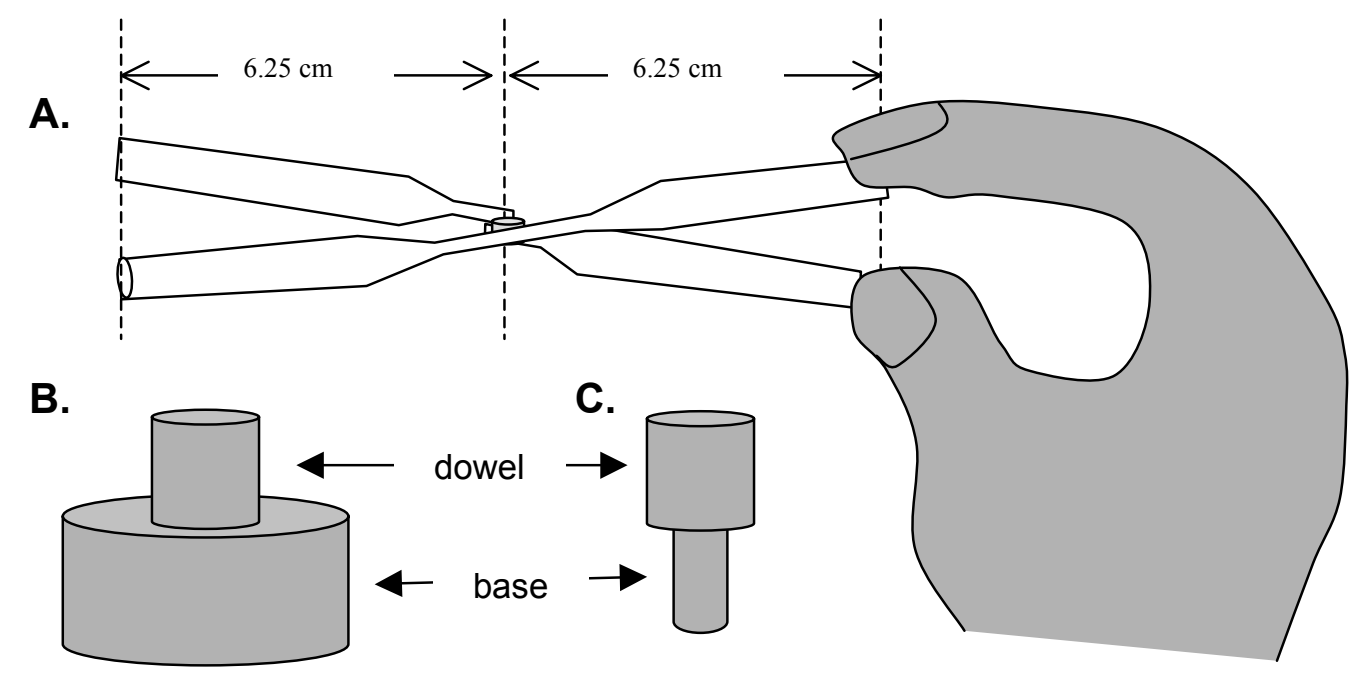

Figure 1. The grasper (A) and the object used for the study. A dowel is placed on a wide $(B)$ or a narrow base $(C)$

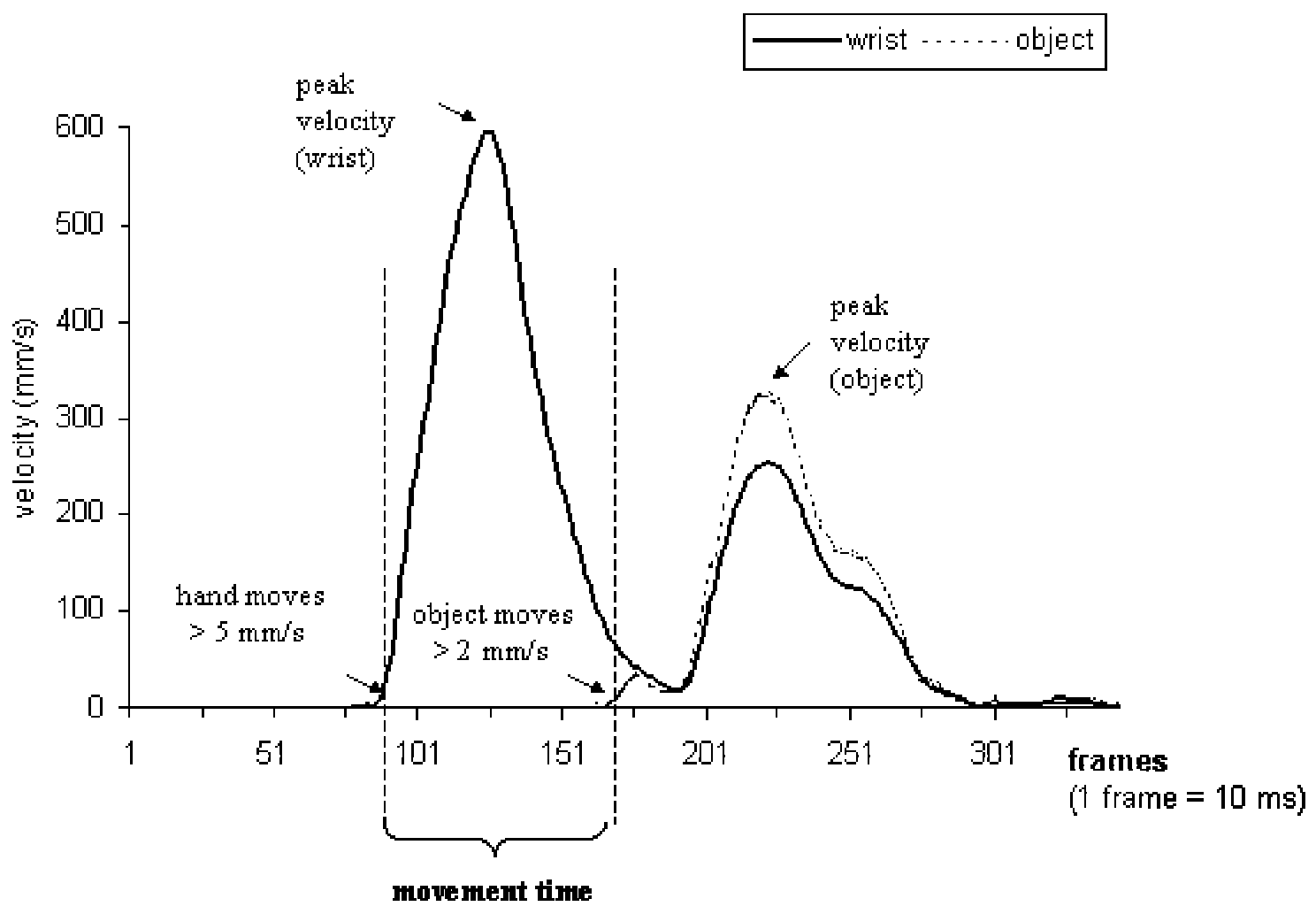

Figure 2. Tangential velocity profiles of the wrist and the object movements. The first occurrence of nonrepeating wrist velocity larger than $5 \mathrm{~mm} / \mathrm{s}$ was defined as the start of the movement; the first occurrence of object movement speed larger than $2 \mathrm{~mm} / \mathrm{s}$ was defined as the end of prehensile movement. 
Table 1 Summary of means and standard errors over experimental conditions in natural and remote prehension.

\begin{tabular}{|c|c|c|c|c|c|c|c|c|c|}
\hline \multirow{3}{*}{\multicolumn{2}{|c|}{$\begin{array}{r}\text { Task } \\
\text { Hand } \\
\text { Object Base }\end{array}$}} & \multicolumn{4}{|c|}{ Natural Prehension } & \multicolumn{4}{|c|}{ Remote Prehension } \\
\hline & & \multicolumn{2}{|c|}{ preferred } & \multicolumn{2}{|c|}{ nonpreferred } & \multicolumn{2}{|c|}{ preferred } & \multicolumn{2}{|c|}{ nonpreferred } \\
\hline & & wide & narrow & wide & narrow & wide & narrow & wide & narrow \\
\hline \multirow[t]{2}{*}{$\mathrm{MT}(\mathrm{ms})$} & mean & 735 & 746 & 811 & 838 & 908 & 926 & 948 & 1044 \\
\hline & std. error & 39 & 44 & 34 & 45 & 59 & 48 & 34 & 37 \\
\hline \multirow[t]{2}{*}{$\mathrm{PV}(\mathrm{mm} / \mathrm{s})$} & mean & 644 & 639 & 607 & 601 & 538 & 528 & 540 & 506 \\
\hline & std. error & 23 & 27 & 20 & 26 & 21 & 14 & 24 & 16 \\
\hline \multirow[t]{2}{*}{ TPV (ms) } & mean & 363 & 369 & 364 & 379 & 430 & 420 & 408 & 424 \\
\hline & std. error & 12 & 13 & 11 & 16 & 24 & 15 & 14 & 12 \\
\hline \multirow[t]{2}{*}{ TAPV (ms) } & mean & 372 & 377 & 447 & 459 & 478 & 506 & 540 & 620 \\
\hline & std. error & 33 & 37 & 30 & 35 & 38 & 38 & 28 & 31 \\
\hline \multirow[t]{2}{*}{$\%$ TAPV } & mean & 49 & 49 & 54 & 54 & 52 & 54 & 56 & 59 \\
\hline & std. error & 2 & 2 & 2 & 2 & 1 & 2 & 1 & 1 \\
\hline \multirow[t]{2}{*}{$\mathrm{PAc}(\mathrm{mm} / \mathrm{s} / \mathrm{s})$} & mean & 3561 & 3461 & 3334 & 3274 & 2443 & 2423 & 2759 & 2242 \\
\hline & std. error & 275 & 297 & 270 & 329 & 195 & 143 & 390 & 117 \\
\hline \multirow[t]{2}{*}{ TPAc (ms) } & mean & 200 & 203 & 192 & 205 & 220 & 224 & 212 & 213 \\
\hline & std. error & 10 & 8 & 8 & 12 & 14 & 10 & 10 & 8 \\
\hline \multirow[t]{2}{*}{$\% \mathrm{TPAc}$} & mean & 28 & 28 & 24 & 25 & 25 & 25 & 23 & 21 \\
\hline & std. error & 2 & 2 & 1 & 2 & 1 & 1 & 1 & 1 \\
\hline \multirow[t]{2}{*}{$\mathrm{PDc}(\mathrm{mm} / \mathrm{s} / \mathrm{s})$} & mean & -2348 & -2291 & -2011 & -1978 & -1776 & -1677 & -2203 & -1571 \\
\hline & std. error & 190 & 202 & 142 & 162 & 136 & 97 & 578 & 95 \\
\hline \multirow[t]{2}{*}{ TPDc (ms) } & mean & 504 & 520 & 524 & 538 & 598 & 589 & 584 & 608 \\
\hline & std. error & 20 & 22 & 18 & 25 & 32 & 24 & 22 & 19 \\
\hline \multirow[t]{2}{*}{$\%$ TPDC } & mean & 70 & 72 & 66 & 66 & 67 & 65 & 62 & 59 \\
\hline & std. error & 3 & 3 & 2 & 2 & 2 & 2 & 2 & 2 \\
\hline \multirow[t]{2}{*}{$\mathrm{PA}(\mathrm{mm})$} & mean & 46 & 52 & 52 & 54 & 57 & 57 & 54 & 57 \\
\hline & std. error & 2 & 2 & 3 & 2 & 2 & 2 & 1 & 2 \\
\hline \multirow[t]{2}{*}{ TPA (ms) } & mean & 635 & 635 & 647 & 651 & 722 & 758 & 746 & 806 \\
\hline & std. error & 32 & 34 & 28 & 33 & 42 & 31 & 35 & 41 \\
\hline \multirow[t]{2}{*}{$\%$ ТРА } & mean & 87 & 86 & 81 & 79 & 81 & 83 & 80 & 78 \\
\hline & std. error & 2 & 2 & 2 & 3 & 3 & 3 & 3 & 3 \\
\hline \multirow[t]{2}{*}{ IID } & mean & 24 & 25 & 27 & 27 & 24 & 24 & 24 & 24 \\
\hline & std. error & 2 & 2 & 2 & 2 & 1 & 1 & 1 & 1 \\
\hline \multirow[t]{2}{*}{$\operatorname{adjPA}$} & mean & 22 & 27 & 25 & 27 & 33 & 33 & 30 & 33 \\
\hline & std. error & 2 & 2 & 3 & 2 & 2 & 2 & 1 & 1 \\
\hline
\end{tabular}

Abbreviation: MT, movement time; PV, peak velocity; TPV, time to peak velocity;

$\% \mathrm{TAPV}$, percent time after peak velocity; PAc, peak acceleration ; TPAc, time to peak acceleration; $\%$ TPAc, percent time to peak acceleration; PDc, peak deceleration; TPDc, time to peak deceleration $\%$ TPAc, percent time to peak deceleration; PA, peak aperture; TPA, time to peak aperture; \%TPA, percent time to peak aperture; IID, initial IREDs difference; adjPA, adjusted peak aperture 
Movements were tracked with the OPTOTRAK 3020 system. The position data taken by infrared emitting diodes (IREDs) placed on the wrist, thumb and index finger were used. Data were sampled at $100 \mathrm{~Hz}$, interpolated at 4 frames, and filtered at $4 \mathrm{~Hz}$ using the WATSMART program. Figure 2 illustrates the definition of the movement time (MT). MT and other kinematic landmarks were computed, including peak velocity (PV), time to peak velocity (TPV), peak acceleration (PAc), time to PAc (TPAc), peak deceleration (PDc), time to PDc (TPDc), peak aperture (PA), time to peak aperture(TPA). Dependent variables were analysed by a 2 (task) $\mathrm{x} 2$ (hand) $\mathrm{x}$ 2 (base) within subject ANOVA using SPSS 11.0.

\section{RESULTS}

Remote prehension had a longer movement time (956 $\mathrm{ms})$ compared to natural prehension $(783 \mathrm{~ms}, \mathrm{P}<0.001)$. Reaching and grasping the object on the wide base $(888 \mathrm{~ms})$ took longer time compared to the narrow base $(851 \mathrm{~ms}$; $\mathrm{P}=$ 0.004). The preferred hand $(829 \mathrm{~ms})$ moved faster than the non-preferred hand $(910 \mathrm{~ms}, \mathrm{P}=0.003)$. In natural prehension, the base width did not show a major impact on the movement (wide $773 \mathrm{~ms}$, narrow $792 \mathrm{~ms}$ ); however, in remote prehension the effects of base width were amplified (wide $928 \mathrm{~ms}$, narrow $985 \mathrm{~ms} ; \mathrm{P}=0.017$ ).

The longer movement time of remote prehension was accompanied by a low movement speed. Peak velocity in remote prehension $(528 \mathrm{~mm} / \mathrm{s})$ was significant lower than in natural prehension $(623 \mathrm{~mm} / \mathrm{s}, \mathrm{P}<0.001)$. Reaching and grasping an object on the narrow base resulted in lower movement speed $(569 \mathrm{~mm} / \mathrm{s})$ compared to the wide base (582 $\mathrm{mm} / \mathrm{s}, \mathrm{P}=0.028)$. Action by the preferred hand $(587 \mathrm{~mm} / \mathrm{s})$ achieved a higher movement speed than the nonpreferred hand $(563 \mathrm{~mm} / \mathrm{s}, \mathrm{p}=0.003)$.

In natural prehension, maximum movement speeds were achieved earlier $(\mathrm{TPV}=369 \mathrm{~ms})$ than remote prehension $(\mathrm{TPV}=421 \mathrm{~ms}, \mathrm{P}=0.002)$. Reaching for an object on the narrow base yielded a longer deceleration phase (TAPV $=491$ $\mathrm{ms}, \% \mathrm{TAPV}=54 \%$ ) than a wide-base object ( $459 \mathrm{~ms}, 53 \%$ ). In natural prehension, the deceleration phase was not significantly influenced by the base width (TAPV of wide base $409 \mathrm{~ms}$, narrow $418 \mathrm{~ms}$ ). However, holding a grasper in the hand significantly extended the deceleration phase when reaching for an object on the narrow base $(\mathrm{TAPV}=563 \mathrm{~ms})$ compared to the wide base (TAPV $=509 \mathrm{~ms})$. In other words, holding a grasper in hand amplified the effect of task precision requirements on movement performance.

The aperture analysis revealed a larger PA in remote (56 $\mathrm{mm})$ than natural prehension $(51 \mathrm{~mm} ; \mathrm{P}=0.016)$. One might argue that the larger PA in the remote task was due to the relatively larger initial aperture of the hand when the tool was attached to the thumb and index finger. The initial aperture was then computed prior to movement start and was subtracted from aperture profiles. The adjusted peak aperture (adjPA) measures the actual increments of the aperture during the movements.

Again, a larger adjPA was observed in remote $(32 \mathrm{~mm})$ than natural prehension $(25 \mathrm{~mm} ; \mathrm{P}=0.002)$. The adjPA was affected significantly by the base width (narrow base $=30$ $\mathrm{mm}$, wide base $=28 \mathrm{~mm}, \mathrm{P}=0.006$ ). A larger proportion of time was used to reach the peak aperture in natural $(83 \%)$ than remote prehension $\left(80 \% ; \mathrm{F}_{1,11}=7.37, \mathrm{p}=0.020\right)$. In other words, a larger percentage of time was used for closing the hand in remote prehension compared to natural prehension.

The cross-correlation between the measure of time to peak deceleration and the time to peak aperture was computed. Suggested by Jeannerod, these two were the key variables for measuring the temporal coupling of reaching and grasping of a prehension(Jeannerod, 1981, 1984). Correlations between the TPDc and TPA were diverse, only 35 of the 96 (36.5\%) correlations were significant. No noteworthy pattern could be found when examining the distribution of these correlations over experimental conditions.

\section{DISCUSSION}

Clear kinematic features of remote prehension included longer movement time, lower movement speed, longer deceleration phase and relatively larger peak aperture compared to the natural prehension. We believe the kinematic changes in remote prehension are caused by difficulties in sensory perception and the sensorimotor integration of the movement.

In remote prehension, proprioception is indirect or incomplete, and vision becomes the primary source for perceiving information of the tool. As a result, visual coding and decoding is more complicated in remote than natural manipulation. In the case of performing a difficult task (higher precision requirement), the volume of information transported along a single visuomotor pathway may increase extensively and reach the maximal capacity. The decrease of movement speed and prolonged movement time allows more time to process information. The velocity profile in the case of escalating requirement for sensorimotor mapping will display a unique skew, i.e. significantly longer movement time during the deceleration phase. For example, Marteniuk \& MacKenzie identified a longer deceleration phase on the course of reaching and grasping a more fragile object (light bulk) compared to a tennis ball (Marteniuk, MacKenzie, Jeannerod, Athenes, \& Dugas, 1987). In this study, deceleration phase for remote prehension was $536 \mathrm{~ms}, 122 \mathrm{~ms}$ longer than natural prehension (414 ms), reflecting the degree of perception difficulty in remote prehension.

Difficulty in obtaining sensory feedback also affects the grasping component. Subjects tended to open their hand wider in remote prehension to prevent from missing the object. In the mean time, subjects reduced movement speed and allowed more time to close the grasper prior to the contact of the object.

A lower correlation coefficient between TPDc and TPA and no noteworthy correlation pattern for natural and remote prehension rejects the notion of temporal coupling between the transport and grasp component. It would be more logical to describe the coordination as a central blueprint that regulates the functional output of each sub-movement to ensure the task goal be accomplished, rather than set rigid couplings on a temporal or spatial aspect (Marteniuk et al., 1990).

This study has implications for design of tools and a safe work environment for tool use. In research and development 
of remote manipulation tools, these kinematic measures like peak velocity, time in deceleration, and peak aperture may be indicators of tool effectiveness for a given task. Further, as we pointed out earlier, the vision becomes extremely important for perceiving information of the tool. A safe work place should provide sufficient illumination when a worker's tasks are performed with tools. In addition, performing with a tool requires extra effort from human users to process information, which suggests a longer time will be allowed for task with tools.

The grasper used in the study was attached to the fingers by tape. This unusual way of holding the tool may limit the generalizability of our findings. Also, the aperture profile that described the movement of the grasp component was measured from the fingertips. Future study on remote manipulation will allow subjects to grip and grasp in a more comfortable way, and grasping outcomes would be detected from both the hands and the tips of these types of graspers.

\section{ACKNOWLEDGEMENTS:}

This research was supported by doctoral fellowships to B. Zheng from Simon Fraser University and Michael Smith Foundation for Health Research, and by research grants to C. L. MacKenzie by the Natural Sciences and Engineering Research Council of Canada.

\section{REFERENCES:}

Driver, J., \& Spence, C. (2000). Multisensory perception: Beyond modularity and convergence. Current Biology, 10(20), R731-735.

Elliott, D., Roy, E. A., \& Goodman, D. (1993). Asymmetries in the preparation and control of manual aiming movements. Canadian Journal of Experimental Psychology, 47(3), 570-589.

Jeannerod, M. (1981). Intersegmental coordination during reaching at natural visual objects. In J. B. Long \& A. Baddeley (Eds.), Attention and performance ix.Hillsdale, New Jersey: Lawrence Erlbaum Associates.

Jeannerod, M. (1984). The timing of natural prehension movements. Journal of Motor Behavior, 16(3), 235-254.

Jeannerod, M. (1988). The neural and behavioural organization of goal-directed movements.New York: Clarendon Press.

Maravita, A., Spence, C., \& Driver, J. (2003). Multisensory integration and the body schema: Close to hand and within reach. Current Biology, 13(13), R531-539.

Maravita, A., Spence, C., Kennett, S., \& Driver, J. (2002). Tool-use changes multimodal spatial interactions between vision and touch in normal humans. Cognition, 83(2), B25-34.

Marteniuk, R. G., Leavitt, J. L., \& MacKenzie, C. L. (1990). Functional relationships between grasp and transport components in a prehension task. Human Movement Science, 9(2), 149-176.
Marteniuk, R. G., MacKenzie, C. L., Jeannerod, M., Athenes, S., \& Dugas, C. (1987). Constraints on human arm movement trajectories. Canadian Journal of Psychology, 41(3), 365-378.

Rothwell, J. C., Traub, M. M., Day, B. L., Obeso, J. A., Thomas, P. K., \& Marsden, C. D. (1982). Manual motor performance in a deafferented man. Brain, 105 (Pt 3), 515-542.

Taub, E. (1976). Movement in nonhuman primates deprived of somatosensory feedback. Exercise and Sport Sciences Reviews, 4, 335-374.

Wing, A. M., Turton, A., \& Fraser, C. (1986). Grasp size and accuracy of approach in reaching. Journal of Motor Behavior, 18(3), 245-260. 